\title{
Mapping Chemistry, Composition, and Dynamics with Coherent Raman Imaging
}

\author{
Marcus T Cicerone ${ }^{1}$, Charles H Camp Jr${ }^{1}$, Ronit Sharon-Frilling ${ }^{1}$ and Young Jong Lee ${ }^{1}$ \\ 1. National Institute of Standards and Technology, Materials Measurements Lab, Gaithersburg, MD, \\ USA
}

We first introduced spectroscopic coherent Raman imaging in 2004 [1], demonstrating good chemical discrimination of bulk polymer blends on a broadband coherent Raman scattering (BCARS) microscope. The good spatial resolution (lateral $300 \mathrm{~nm}$ and axial $1 \mu \mathrm{m}$ ) and resolution and short spectral integration times $(17 \mathrm{~ms})$ portended utility in materials and biological imaging. However, the CARS signal is proportional to the square of the local analyte concentration, so the early instrumentation was not able to acquire spectra from weakly scattering and relatively low concentration species represented in the fingerprint region of the spectrum.

After many improvements in instrumentation and signal retrieval methods it is now possible to obtain quantitative and robust fingerprint and $\mathrm{CH}$-stretch spectra from materials and biological systems. The BCARS signal is generated by combining a spectrally narrow probe field with a spectrally broad Stokes field. The resulting signal includes a component that is resonant with molecular vibrations, related to the spontaneous Raman signal, and a field that is not resonant, and nominally frequency independent. The latter can be much stronger than the former, and we found that it masked the weak fingerprint spectra. One of the most important advances was the realization that the nonresonant component of the coherent signal could be used to amplify the weak resonant component of interest [2], and once baseline was removed [3], it was possible to obtain quantitative and reliable spectra from materials [4] and cells [5]. Subsequent correction to errors in nonresonant background estimation [6] ensured reproducible spectra with internally calibrated peak ratios. With these improvements it was possible to obtain good quality spectra from biological cells in the $\mathrm{CH}$ stretch region, but still only marginal fingerprint spectral peaks.

The early BCARS instrumentation relied on a continuum Stokes field that was intrinsically noise-prone, and that noise was transferred to the anti-Stokes signal, obscuring weak fingerprint peaks. Optimizing the continuum [7] was one key to improving the signal, but ultimately, an entirely different type of supercontinuum pulse that was temporally compressible to $15 \mathrm{fs}$, allowed us to utilize a second and more efficient signal generation mechanism. Together, these factors lead to acquisition of even weak fingerprint spectra from biological tissues in approximately $3 \mathrm{~ms}$ [8]. The resulting state-of-the-art spectroscopic coherent Raman imaging (CRI) instrument provides rapid, high spatial resolution imaging with excellent label-free chemical contrast as shown in Figure 1. It has allowed us to shed light on phenomena in biology and materials science. Examples in biology include mechanisms of intercellular signaling [9], and adhesion strategies of mollusks [10]. We have also been able to use this imaging modality to chemically map pharmaceutical preparations, finding rare, but potentially important features in drug phase that were not visible to spontaneous Raman imaging due to their small size [11].

In addition to chemical contrast, it appears that it will be possible to derive a contrast mechanism based on materials dynamics from CRI methods. Recent advances in our understanding of motion in solids allows us to predict dynamic properties of these systems over 12 orders of magnitude in time with just a few parameters derived from picosecond timescale measurements [12]. We have previously shown that these ps dynamics can be used to predict stability of proteins in pharmaceutical preparations [13], and it 
has recently been shown that they are also related to crystallization rate of small molecule drugs [14]. I will show that coherent Raman techniques can provide the parameters needed to predict dynamic properties, making it possible to spatially map materials dynamics in a molecularly specific way, and providing spatially resolved information on relative protein degradation rates or drug crystallization rates.

References:

[1] T. W. Kee, M. T. Cicerone, Optics Letters 29 (2004), 2701-3.

[2] Y. X. Liu, Y. J. Lee, M. T. Cicerone, Optics Letters 34 (2009), 1363-5.

[3] Y. X. Liu, Y. J. Lee, M. T. Cicerone, Journal of Raman Spectroscopy 40 (2009), 726-31.

[4] Y. J. Lee, D. Moon, et al., Anal Chem 83 (2011), 2733-9.

[5] S. H. Parekh, Y. J. Lee, et al., Biophysical Journal 99 (2010), 2695-704.

[6] C. H. Camp, Y. J. Lee, M. T. Cicerone, Journal of Raman Spectroscopy (2015), n/a-.

[7] Y. J. Lee, S. H. Parekh, et al., Optics Express 18 (2010), 4371-9.

[8] C. H. Camp Jr, Y. J. Lee, et al., Nat Photon 8 (2014), 627-34.

[9] P. V. Afonso, M. Janka-Junttila, et al., Dev Cell 22 (2012), 1079-91.

[10] N. V. Gohad, N. Aldred, et al., Nat Commun 5 (2014).

[11] C. M. Hartshorn, Y. J. Lee, et al., Anal Chem 85 (2013), 8102-11.

[12] M. T. Cicerone, Q. Zhong, M. Tyagi, Physical Review Letters 113 (2014), 117801-.

[13] M. T. Cicerone, J. F. Douglas, Soft Matter 8 (2012), 2983.

[14] J. Sibik, K. Löbmann, et al., Mol Pharm 12 (2015), 3062-8.

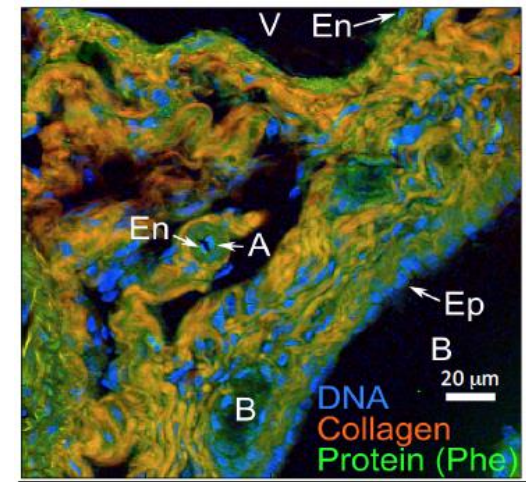

V: Vein; B: Bile Duct; A: Artery; L: Lipid Droplet; En: Endothelial Cell; Ep: Epithelial Cell.

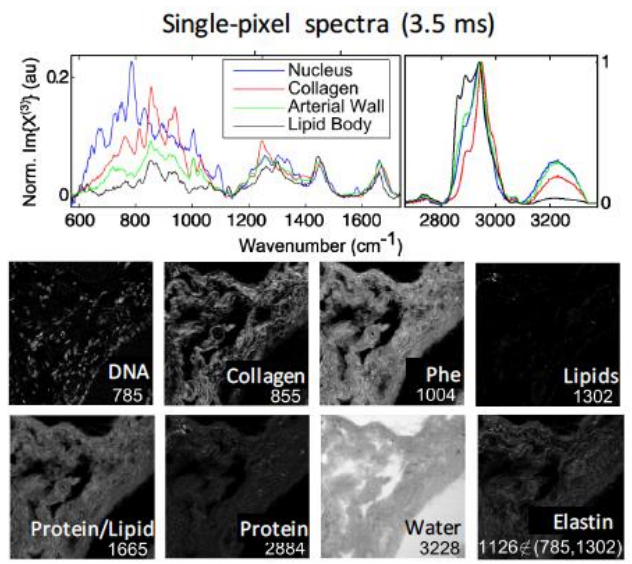

Figure 1. Murine liver section imaged with broadband coherent Raman scattering microscopy. Contrast in micrographs is derived from Raman spectra contained at each image pixel.

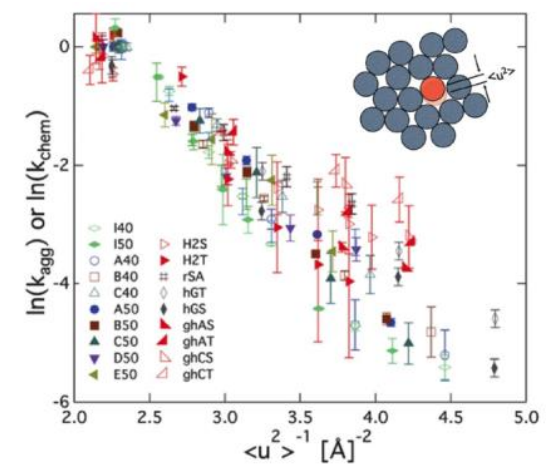

Figure 2. Chemical and physical degradation rates for proteins dried in various sugar-based formulations and stored under various stress conditions, plotted against mean squared displacement in protein / sugar matrix. Each symbol represents a separate formulation or stress condition, and these are explained in reference [13] 